\title{
Proteomic Analysis of Cardioembolic and Large Artery Atherosclerotic Clots Using Reverse Phase Protein Array Technology Reveals Key Cellular Interactions Within Clot Microenvironments
}

Mehdi Abbasi ${ }^{1}$, Sean Fitzgerald ${ }^{2}$, Jennifer Ayers-Ringler ${ }^{1}$, Virginia Espina ${ }^{3}$, Claudius Mueller ${ }^{3}$, Sally Rucker $^{3}$, Ramanathan Kadirvel ${ }^{1}$, David Kallmes ${ }^{1}$, Waleed Brinjikji ${ }^{4}$

1. Radiology, Mayo Clinic, Rochester, USA 2. Physiology, National University of Ireland Galway, Galway, IRL 3. Center for Applied Proteomics and Molecular Medicine, George Mason University, Manassas, USA 4. Neuroradiology, Mayo Clinic, Rochester, USA

Corresponding author: Mehdi Abbasi, abbasi.mehdi@mayo.edu

\begin{abstract}
Thrombus characteristics are dependent on clot composition, but identification of the etiology based on histological analysis has proved inconclusive. Identification of proteomic signatures may help to differentiate between clots of different etiologies such as cardioembolic, large artery atherosclerotic, and other known etiologies, information that could enhance an individualized medicine approach to secondary stroke prevention. In this study, total protein extracts from cardioembolic $(n=25)$ and large artery atherosclerotic $(n=23)$ thrombus specimens were arrayed in quadruplicate on nitrocellulose slides and immunostained for 31 proteins using a Dako Autostainer (Agilent Technologies, Inc., Santa Clara, USA). We quantified 31 proteins involved in platelet and/or endothelial function, inflammation, oxidative stress, and metabolism. Pathway analysis showed more heterogeneity and protein network interactions in the cardioembolic clots but no specific correlations with clot etiology. Reverse-phase protein arrays are a powerful tool for assessing cellular interactions within the clot microenvironment and may enhance understanding of clot formation and origination. This tool could be further explored to help in identifying stroke etiology in large vessel occlusion patients with embolic stroke of an undetermined source.
\end{abstract}

Categories: Neurology, Pathology

Keywords: endocannabinoid, inflammation, phosphoprotein, proteomics, thrombus, stroke, thrombectomy

\section{Introduction}

Ischemic stroke is caused by a thromboembolic occlusion of cerebral arteries. Treatment is focused on fast and efficient removal of the occluding clot with either intravenous thrombolysis or with endovascular thrombectomy. Although the occluding clot is the primary target of treatment, little is known about its internal organization and inflammatory or cytokines that are involved in the formation or evolution of clots. Also, cellular pathways and cells governing the structure of clots has become a hot topic in the medical literature. A recent subgroup analysis from the Multicenter Randomized Clinical Trial of Endovascular Treatment for Acute Ischemic Stroke in the Netherlands (MR CLEAN) Registry has confirmed that thrombus computed tomography characteristics of cardioembolic (CE) stroke are distinct from those of non-CE stroke. Non-CE strokes were associated with the presence of hyperdense artery sign, a higher clot burden, a shift towards a more proximal thrombus location, and longer thrombi compared with a CE cause [1]. The differences in imaging characteristics between stroke etiologies are likely a result of differences in the composition of the occlusive thrombus resulting from dissimilarities in the clotting mechanisms. Histological analysis can reveal microscopic clot composition, but studies relating etiology to clot composition have thus far proved inconclusive [2]. Identification of proteomic signatures of thrombi from different stroke etiologies may prove invaluable in identifying the true etiology of the roughly $40 \%$ of ischemic stroke patients that have no known cause, which complicates effective secondary stroke prevention in these patients [3]. This study sought to investigate the proteomic signature of CE and large artery atherosclerotic (LAA) clots.

\section{Materials And Methods}

\section{Patients}

All patients underwent comprehensive neurovascular imaging for evaluation of the presence of carotid atherosclerotic disease, including CT angiography from the aorta to the intracranial arterial vasculature. All patients also underwent a standard cardiac evaluation, including a transthoracic echocardiogram (TTE)/transesophageal echocardiogram (TEE), electrocardiogram (EKG). In cases in which no abnormality was found on the initial cardiac workup, prolonged cardiac monitoring was performed using either a Holter monitor or implantable cardiac monitoring device. Patients were categorized according to Toast criteria [4]. The study was approved by the Mayo Clinic Institutional Review Board, and a waiver of consent was granted. 


\section{Cureus}

\section{Specimens}

Thrombus specimens were collected from patients undergoing mechanical thrombectomy at Mayo Clinic between October 2016 and July 2019 (Table 1$)$. The CE $(n=25)$ and LAA $(n=23)$ thrombus specimens were fixed in $10 \%$ neutral buffered formalin for up to 24 hours and processed into paraffin blocks (FFPE).

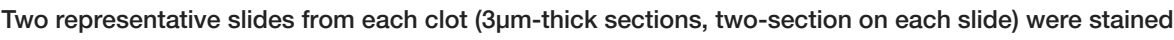
with Martius Scarlett Blue stain. After regular histological staining, three $50 \mu \mathrm{m}$ sections were cut from each FFPE block, placed into a microcentrifuge tube, and stored at $4^{\circ} \mathrm{C}$. Two tissue sections from each specimen were deparaffinized in the tube using two exchanges of xylene (three minutes at $50^{\circ} \mathrm{C}$ ). The tissue sections were rehydrated in graded ethanol $(100 \%, 95 \%, 70 \%$, water with protease inhibitors (Roche cOmplete $\left.{ }^{\mathrm{TM}} \mathrm{Mini}\right)$, and lysed in protein lysis buffer $(450 \mu \mathrm{L}$ tissue protein extraction reagent

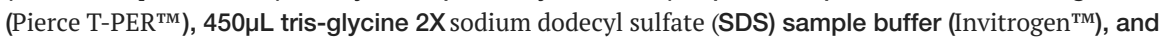
$100 \mu \mathrm{L}$ tris(2-carboxyethyl)phosphine (Pierce ${ }^{\mathrm{TM}} \mathrm{TCEP}$ Solution, neutral $\mathrm{pH}$, Bond-Breaker ${ }^{\mathrm{TM}}$ ). Tissue lysates were heated at $95^{\circ} \mathrm{C}$ for eight minutes and stored at $-80^{\circ} \mathrm{C}$.

\begin{tabular}{|c|c|c|}
\hline & LAA group, $n(\mathrm{~N}=23)$ & Cardioembolic group, $n(N=25)$ \\
\hline Age (years), mean $( \pm \mathrm{SD})$ & $68( \pm 11)$ & $69( \pm 13)$ \\
\hline Gender (male) & 15 & 12 \\
\hline NIHSS at admission, mean $( \pm$ SD) & $17( \pm 6)$ & $17( \pm 7)$ \\
\hline Diabetes & 5 & 4 \\
\hline Hypertension & 10 & 18 \\
\hline Dyslipidemia & 12 & 15 \\
\hline Smoking & 1 & 4 \\
\hline \multicolumn{3}{|l|}{ Occlusion location } \\
\hline ICA & 5 & 7 \\
\hline ICA terminus & 2 & 4 \\
\hline M1 & 11 & 13 \\
\hline M2 & 2 & 3 \\
\hline M3 & 1 & 0 \\
\hline Vertebral & 1 & 0 \\
\hline Basilar & 1 & 0 \\
\hline P1 & 0 & 1 \\
\hline rTPA technique & 10 & 13 \\
\hline Aspiration & 16 & 17 \\
\hline Stentretriever & 2 & 3 \\
\hline Combination & 5 & 5 \\
\hline No of passes, mean ( $\pm \mathrm{SD}$ ) & $2.2( \pm 1.3)$ & $2( \pm 1)$ \\
\hline Final TICl score (2b or higher) & 21 & 23 \\
\hline
\end{tabular}

\section{TABLE 1: Baseline characteristics of patients}

NIHSS - National Institutes of Health Stroke Scale; ICA - internal carotid artery; rTPA - recombinant tissue plasminogen activator; LAA - large artery atherosclerotic; $\mathrm{TICl}$ - thrombolysis in cerebral infarction

\section{Reverse phase protein arrays (RPPA)}

Thrombus lysates were printed in technical quadruplicates onto nitrocellulose coated glass slides (ONCYTE ${ }^{\circledR}$ Avid, Grace Bio-Labs, Bend, USA), in serial 2-fold dilution curves, using a Quanterix ${ }^{\mathrm{TM}} 2470$ arrayer (Billerica, USA) equipped with 350 $\mu \mathrm{m}$ solid pins (Figure 1). EB1 (BD 611546, Burkitt's lymphoma), 
MOLT-4 (Santa Cruz Biotechnology sc-2233, acute lymphoblastic leukemia), normal umbilical vein tissue (Abcam ab29585), and Jurkat+Calyculin (Santa Cruz Biotechnology sc-2277, human acute T cell leukemia induced with Calyculin A cell lysates) were printed on each array as quality control samples. Bovine serum albumin (BSA) was printed in an 8 point, 2-fold dilution series, starting at $1.0 \mathrm{mg} / \mathrm{mL}$ as a calibration curve for total protein $[5,6]$. The total protein within each array spot was determined using SYPRO ${ }^{\circledR}$ Ruby protein blot stain /Invitrogen ${ }^{\mathrm{TM}}$ Molecular Probes ${ }^{\mathrm{TM}}$ ) per manufacturer's directions and scanned using a Cy3 laser (PowerScanner ${ }^{\mathrm{TM}}$, Tecan Group Ltd., Männedorf, Switzerland). Immunostaining for 31 proteins was performed on a Dako Autostainer per the manufacturer's instructions (CSA kit, Dako, Agilent Technologies, Inc., Santa Clara, USA). Each slide was incubated with a single primary antibody at room temperature for 30 minutes (Table 2). Antibody specificity was confirmed by Western blotting as previously described [7]. The negative control slide was incubated with antibody diluent only. The secondary antibody was goat antirabbit immunoglobulin G (IgG) H+L (1:10,000; Vector Labs, Burlingame, USA). Signal detection was amplified via horseradish peroxidase mediated biotinyl tyramide deposition with chromogenic detection (diaminobenzidine) per manufacturer's instructions (Dako, Agilent Technologies, Inc., Santa Clara, USA). Arrays were scanned at 600dpi on a flatbed scanner (PowerLook, UMAX ${ }^{\circledR}$, Dallas, USA). Spot (pixel) intensity was analyzed using ImageQuant ${ }^{\mathrm{TM}}$ ver. 5.2 (GE Healthcare, Chicago, USA), with mean local area background subtraction. Signal intensity for each spot was calculated using the freely available data reduction algorithm (RAS ver. 16) [5]. Spot intensities were normalized to total protein/spot.

\begin{tabular}{|c|c|c|c|c|}
\hline RPPA protein & Full name & Company & $\begin{array}{l}\text { Catalog } \\
\#\end{array}$ & Dilution \\
\hline AcCoA Ser79 & Acetyl-CoA carboxylase phospho Ser79 & $\begin{array}{l}\text { Cell Signaling } \\
\text { Technology }\end{array}$ & 3661 & 1:1000 \\
\hline Androgen receptor & Androgen receptor & $\begin{array}{l}\text { Cell Signaling } \\
\text { Technology }\end{array}$ & 5153 & $1: 100$ \\
\hline Arginase-1 & Arginase-1 & $\begin{array}{l}\text { Cell Signaling } \\
\text { Technology }\end{array}$ & 9819 & 1:100 \\
\hline Biliverdin reductase & Biliverdin reductase & Assay Designs & OSA-400 & $1: 750$ \\
\hline CD234/DARC & Duffy antigen chemokine receptor & Pierce & $\begin{array}{l}\text { PA5- } \\
18424\end{array}$ & $1: 50$ \\
\hline CD63 & CD63 & Abcam & ab134045 & 1:1000 \\
\hline CD45 & Protein tyrosine phosphatase receptor type C & BD & 610265 & $1: 200$ \\
\hline CD79a & $\begin{array}{l}\text { B-cell antigen receptor complex-associated protein alpha } \\
\text { chain }\end{array}$ & $\begin{array}{l}\text { Cell Signaling } \\
\text { Technology }\end{array}$ & 13333 & $1: 100$ \\
\hline $\begin{array}{l}\text { Cannabinoid receptor I } \\
\text { (CNR1, CB1) }\end{array}$ & Cannabinoid receptor 1 & Pierce & PA1-745 & $1: 50$ \\
\hline $\begin{array}{l}\text { Cannabinoid receptor II } \\
\text { (CNR2, CB2) }\end{array}$ & Cannabinoid receptor 2 & Pierce & $\begin{array}{l}\text { PA1- } \\
746 A\end{array}$ & $1: 100$ \\
\hline Collagen I & Collagen I & $\begin{array}{l}\text { Santa Cruz } \\
\text { Biotechnology }\end{array}$ & sc-80760 & $1: 50$ \\
\hline Collagen IV & Collagen IV & Dako & M0785 & $1: 50$ \\
\hline DAG-lipase beta & Diacylglycerol-lipase beta & $\begin{array}{l}\text { Cell Signaling } \\
\text { Technology }\end{array}$ & 12574 & 1:100 \\
\hline eNOS Ser177 & Endothelial nitric oxide synthase phospho Ser177 & $\begin{array}{l}\text { Cell Signaling } \\
\text { Technology }\end{array}$ & 9571 & 1:50 \\
\hline Galectin-3 & Galectin-3 & $\begin{array}{l}\text { Cell Signaling } \\
\text { Technology }\end{array}$ & 87985 & $1: 300$ \\
\hline GRK2 & $\begin{array}{l}\text { G-protein coupled receptor kinase } 2 / \beta \text {-adrenergic } \\
\text { receptor kinase } 1 \text { ( } \beta \text {-ARK1) }\end{array}$ & $\begin{array}{l}\text { Cell Signaling } \\
\text { Technology }\end{array}$ & 3982 & $1: 250$ \\
\hline IL-6 & Interleukin-6 & BioVision & $5143-100$ & $1: 100$ \\
\hline IL-8 & Interleukin-8 & Abcam & ab7747 & $1: 200$ \\
\hline IL-10 & Interleukin-10 & Abcam & ab52909 & $1: 2000$ \\
\hline
\end{tabular}




\section{Cureus}

\begin{tabular}{|c|c|c|c|c|}
\hline IL-11 & Interleukin-11 & $\begin{array}{l}\text { Santa Cruz } \\
\text { Biotechnology }\end{array}$ & sc-7924 & $1: 500$ \\
\hline Neutrophil elastase & Neutrophil elastase & Abcam & ab68672 & 1:100 \\
\hline PDK-1 Ser241 & $\begin{array}{l}\text { Phosphoinositide-dependent protein kinase } 1 \text { phospho } \\
\text { Ser241 }\end{array}$ & $\begin{array}{l}\text { Cell Signaling } \\
\text { Technology }\end{array}$ & 3061 & 1:200 \\
\hline PKC alpha Ser657 & Protein kinase C a phospho Ser657 & Upstate & 06-822 & 1:1000 \\
\hline $\begin{array}{l}\text { PKC alpha/beta } \\
\text { Thr638/641 }\end{array}$ & Protein kinase $\mathrm{C} \alpha / \beta$ phospho Thr638-641 & $\begin{array}{l}\text { Cell Signaling } \\
\text { Technology }\end{array}$ & 9375 & 1:200 \\
\hline PLC gamma Y783 & $\begin{array}{l}\text { Phosphoinositide-specific phospholipase C phospho } \\
\text { Tyr783 }\end{array}$ & $\begin{array}{l}\text { Cell Signaling } \\
\text { Technology }\end{array}$ & 2821 & 1:100 \\
\hline PP2A & Protein phosphatase $2 \mathrm{~A}$ & $\begin{array}{l}\text { Cell Signaling } \\
\text { Technology }\end{array}$ & 2039 & 1:1000 \\
\hline PPARgamma & Peroxisome proliferator-activated receptor-gamma & $\begin{array}{l}\text { Cell Signaling } \\
\text { Technology }\end{array}$ & 2435 & $1: 50$ \\
\hline Serotonin & Serotonin (5-hydroxytryptophan, 5-HT) & Sigma & S5545 & $1: 1000$ \\
\hline Syk Y525/526 & Spleen tyrosine kinase phospho525/526 & $\begin{array}{l}\text { Cell Signaling } \\
\text { Technology }\end{array}$ & 2711 & $1: 50$ \\
\hline TGF- $\beta$ & Transforming growth factor $\beta$ & $\begin{array}{l}\text { Cell Signaling } \\
\text { Technology }\end{array}$ & 3709 & $1: 2000$ \\
\hline vWF & von Willebrand factor & $\begin{array}{l}\text { Cell Signaling } \\
\text { Technology }\end{array}$ & 55707 & 1:100 \\
\hline
\end{tabular}

TABLE 2: Protein names and antibody information for reverse phase protein arrays RPPA - reverse phase protein arrays 


\section{Cureus}

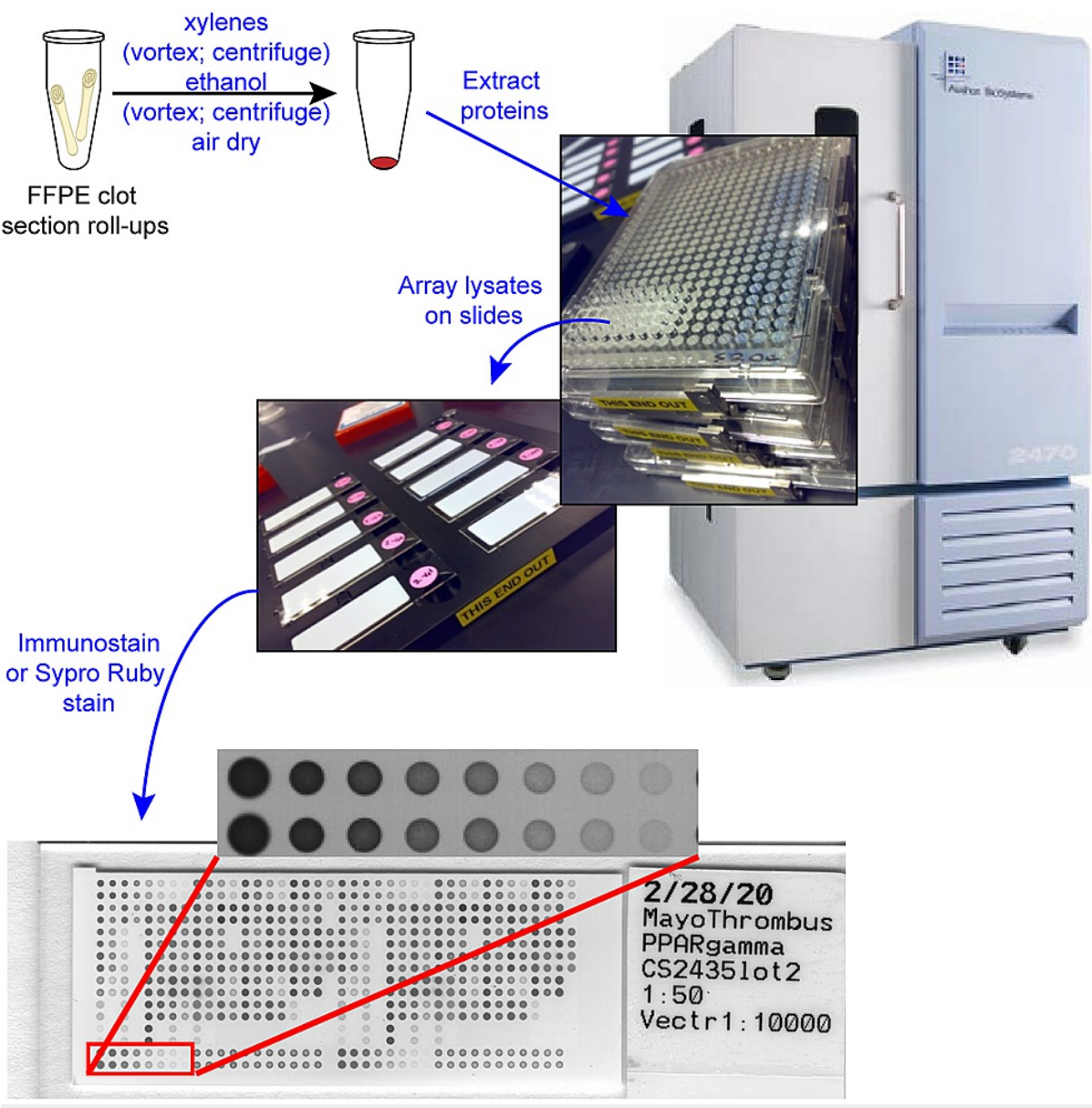

FIGURE 1: Proteomic workflow using formalin-fixed, paraffin-embedded (FFPE) thrombus specimens

Proteins were extracted from FFPE clot specimens, arrayed on nitrocellulose-coated glass slides using a Quanterix 2470 arrayer, and immunostained, or stained for total protein on a Dako Autostainer.

\section{Statistical methods}

Normality was assessed using the Shapiro-Wilk test. Wilcoxon signed-rank sum pairwise comparisons were used for non-normally distributed data with $\mathrm{p} \leqslant 0.05$ considered significant. Data was standardized (z-score) for each protein. Two-way, hierarchical cluster analysis (Euclidean distance, Ward method), correlograms, and Spearman's rho non-parametric correlations were performed in R (R Foundation for Statistical Computing, Vienna, Austria). Spearman's rho non-parametric correlations were used to compare pairs of protein endpoints ( $r h o \geqslant 0.85$ and $\mathrm{p} \leqslant 0.001$ were considered significant). Network diagrams were created in Cytoscape ver. 3.8.0 [8].

\section{Results}

\section{Histological composition of LAA and cardioembolic clots}

The Martius scarlet blue (MSB) histological analysis revealed that there were slight differences in the histological clot composition between the LAA and cardioembolic clots (Figure 2). Cardioembolic clots had a higher proportion of red blood cells (RBCs; $47.67 \%$ vs. $42.58 \%$ ) and white blood cells (WBCs; $4.22 \%$ vs. 3.12\%) compared to LAA clots. On the other hand, LAA clots had a slightly larger proportion of fibrin (31.31\% vs. $29.19 \%)$ and platelets/other (20.81\% vs. $18.21 \%)$. Collagen ( $0.10 \%$ vs. $0.59 \%$ ) and calcification $(0.61 \%$ vs. $1.59 \%)$ are minor components of both cardioembolic and LAA clots respectively. 


\section{Cureus}

\section{MSB Histological Compostion}

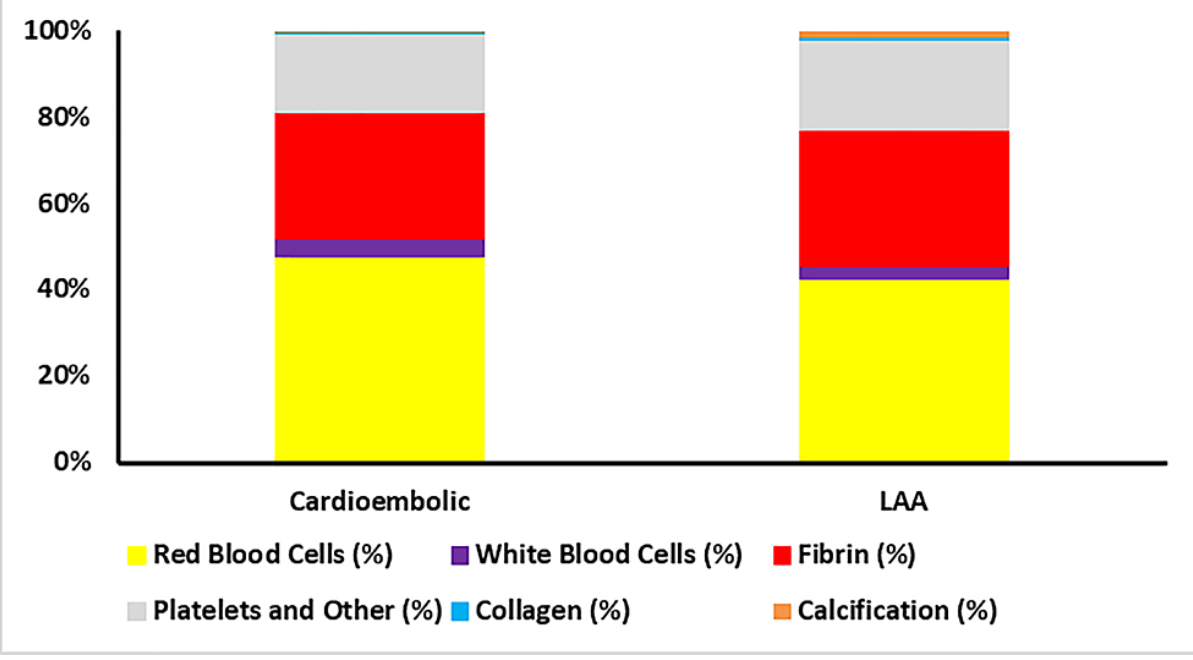

FIGURE 2: Histological clot composition of cardioembolic and LAA clots

The mean histological clot composition (\%) of clot component, as determined by MSB staining represented as the percentage of the total.

MSB - Martius scarlet blue; LAA - large artery atherosclerotic

Proteomic heterogeneity exists within classes of thrombus specimens.

Data for two specimens in the LAA group were not used for hierarchical clustering and network analyses due to missing data (poor precision between technical replicates) for CD63, CD45, and collagen IV. Unsupervised two-way hierarchical clustering revealed two primary clusters of specimens; however, each cluster showed heterogeneity in relation to the etiology of the clot (Figure 3). The protein levels for the 31 proteins in our study could not clearly discriminate CE and LAA thrombus types.



FIGURE 3: Proteomic heterogeneity exists within cardioembolic and large artery atherosclerotic thrombus specimens

Unsupervised hierarchical two-way clustering of the entire cohort of thrombus specimens revealed two heterogeneous clusters of specimens (columns) that contained both CE and LAA thrombus specimens ( $\mathrm{n}=25$ $\mathrm{CE}, \mathrm{n}=21 \mathrm{LAA}$, Euclidean distance, Ward method). The 'variables' dendrogram on the $y$-axis indicates the 


\section{Cureus}

relationships between the expressions of all variables (rows) for all thrombus specimens. The 'variables' concentrations are depicted using a color scale from red (high), to white, to blue (low).

LAA - large artery atherosclerotic; CE - cardioembolic

\section{Cardioembolic clots possess diverse protein signaling interactions}

A pairwise protein linkage analysis was conducted using Spearman's rho non-parametric correlation to map inter-related signaling kinases. Fifty strong protein linkages were noted for the $C E(r h o \geqslant 0.85, p<0.001)$, while only 18 strong linkages were noted for the LAA group (Table 3). No significant negative protein interactions were noted for either group.

\begin{tabular}{|c|c|c|c|c|c|}
\hline $\begin{array}{l}\text { CE protein } \\
\text { variable } 1\end{array}$ & $\begin{array}{l}\text { CE protein } \\
\text { variable } 2\end{array}$ & $\begin{array}{l}\text { Spearman rho_CE } \\
(p<0.001)\end{array}$ & $\begin{array}{l}\text { LAA protein } \\
\text { variable } 1\end{array}$ & $\begin{array}{l}\text { LAA protein } \\
\text { variable } 2\end{array}$ & $\begin{array}{l}\text { Spearman rho_LAA } \\
(p<0.001)\end{array}$ \\
\hline Arginase-1 & IL-10 & 0.88 & & & \\
\hline Arginase-1 & IL-6 & 0.87 & & & \\
\hline Arginase-1 & IL-11 & 0.86 & & & \\
\hline $\begin{array}{l}\text { Cannabinoid Rec } \\
1\end{array}$ & $\begin{array}{l}\text { PKCaß Thr } \\
638 / 641\end{array}$ & 0.90 & & & \\
\hline $\begin{array}{l}\text { Cannabinoid Rec } \\
1\end{array}$ & CD234 (DARC) & 0.87 & & & \\
\hline $\begin{array}{l}\text { Cannabinoid Rec } \\
2\end{array}$ & $\begin{array}{l}\text { Cannabinoid Rec } \\
1\end{array}$ & 0.94 & $\begin{array}{l}\text { Cannabinoid Rec } \\
2\end{array}$ & $\begin{array}{l}\text { Cannabinoid Rec } \\
1\end{array}$ & 0.93 \\
\hline $\begin{array}{l}\text { Cannabinoid Rec } \\
2\end{array}$ & Serotonin & 0.88 & & & \\
\hline $\begin{array}{l}\text { Cannabinoid Rec } \\
2\end{array}$ & $\begin{array}{l}\text { PKCaß Thr } \\
638 / 641\end{array}$ & 0.85 & & & \\
\hline CD63 & $\begin{array}{l}\text { Cannabinoid Rec } \\
1\end{array}$ & 0.95 & & & \\
\hline CD63 & $\begin{array}{l}\text { Cannabinold Rec } \\
2\end{array}$ & 0.90 & & & \\
\hline eNOS Ser177 & DAG Lipase Beta & 0.89 & & & \\
\hline IL-10 & $\begin{array}{l}\text { Biliverdin } \\
\text { Reductase }\end{array}$ & 0.85 & & & \\
\hline IL-6 & $\begin{array}{l}\text { Cannabinoid Rec } \\
1\end{array}$ & 0.97 & & & \\
\hline IL-6 & CD63 & 0.96 & IL-6 & CD63 & 0.89 \\
\hline IL-6 & $\begin{array}{l}\text { Cannabinoid Rec } \\
2\end{array}$ & 0.95 & & & \\
\hline IL-6 & $\begin{array}{l}\text { PKCaß Thr } \\
638 / 641\end{array}$ & 0.88 & & & \\
\hline IL-6 & CD234 (DARC) & 0.88 & & & \\
\hline IL-8 & PKCa Ser657 & 0.96 & IL-8 & PKCa Ser657 & 0.99 \\
\hline $\begin{array}{l}\text { Neutrophil } \\
\text { Elastase }\end{array}$ & IL-6 & 0.98 & $\begin{array}{l}\text { Neutrophil } \\
\text { Elastase }\end{array}$ & IL-6 & 0.94 \\
\hline $\begin{array}{l}\text { Neutrophil } \\
\text { Elastase }\end{array}$ & $\begin{array}{l}\text { Cannabinoid Rec } \\
1\end{array}$ & 0.97 & $\begin{array}{l}\text { Neutrophil } \\
\text { Elastase }\end{array}$ & $\begin{array}{l}\text { Cannabinoid Rec } \\
1\end{array}$ & 0.89 \\
\hline $\begin{array}{l}\text { Neutrophıl } \\
\text { Elastase }\end{array}$ & $\begin{array}{l}\text { Cannabinoid Rec } \\
2\end{array}$ & 0.96 & $\begin{array}{l}\text { Neutrophıl } \\
\text { Elastase }\end{array}$ & $\begin{array}{l}\text { CannabinoId Rec } \\
2\end{array}$ & 0.88 \\
\hline
\end{tabular}




\section{Cureus}

\begin{tabular}{|c|c|c|c|c|c|}
\hline $\begin{array}{l}\text { Neutrophil } \\
\text { Elastase }\end{array}$ & CD63 & 0.94 & & & \\
\hline $\begin{array}{l}\text { Neutrophil } \\
\text { Elastase }\end{array}$ & CD234 (DARC) & 0.90 & & & \\
\hline $\begin{array}{l}\text { Neutrophil } \\
\text { Elastase }\end{array}$ & $\begin{array}{l}\text { PKCaß Thr } \\
638 / 641\end{array}$ & 0.87 & & & \\
\hline PDK1 Ser241 & PKCa Ser657 & 0.98 & PDK1 Ser241 & PKCa Ser657 & 0.99 \\
\hline PDK1 Ser241 & IL-8 & 0.95 & PDK1 Ser241 & IL-8 & 0.98 \\
\hline $\begin{array}{l}\text { PLCgamma } \\
\text { Tyr783 }\end{array}$ & $\begin{array}{l}\text { РКСаß Thr } \\
638 / 641\end{array}$ & 0.94 & & & \\
\hline PPARgamma & $\begin{array}{l}\text { Neutrophil } \\
\text { Elastase }\end{array}$ & 0.98 & PPARgamma & $\begin{array}{l}\text { Neutrophil } \\
\text { Elastase }\end{array}$ & 0.91 \\
\hline PPARgamma & IL-6 & 0.98 & PPARgamma & IL-6 & 0.89 \\
\hline PPARgamma & $\begin{array}{l}\text { Cannabinoid Rec } \\
1\end{array}$ & 0.98 & PPARgamma & $\begin{array}{l}\text { Cannabinoid Rec } \\
1\end{array}$ & 0.95 \\
\hline PPARgamma & Syk Tyr525/526 & 0.97 & PPARgamma & Syk Tyr525/526 & 0.93 \\
\hline PPARgamma & $\begin{array}{l}\text { Cannabinoid Rec } \\
2\end{array}$ & 0.95 & PPARgamma & $\begin{array}{l}\text { Cannabinoid Rec } \\
2\end{array}$ & 0.86 \\
\hline PPARgamma & CD63 & 0.94 & & & \\
\hline PPARgamma & vWF & 0.90 & & & \\
\hline PPARgamma & $\begin{array}{l}\text { РКСaß Thr } \\
638 / 641\end{array}$ & 0.89 & & & \\
\hline PPARgamma & CD234 (DARC) & 0.88 & & & \\
\hline PPARgamma & Arginase-1 & 0.85 & & & \\
\hline Syk Tyr525/526 & $\begin{array}{l}\text { Cannabinoid Rec } \\
1\end{array}$ & 0.97 & Syk Tyr525/526 & $\begin{array}{l}\text { Cannabinoid Rec } \\
1\end{array}$ & 0.91 \\
\hline Syk Tyr525/526 & IL-6 & 0.96 & Syk Tyr525/526 & IL-6 & 0.92 \\
\hline Syk Tyr525/526 & $\begin{array}{l}\text { Neutrophil } \\
\text { Elastase }\end{array}$ & 0.95 & Syk Tyr525/526 & $\begin{array}{l}\text { Neutrophil } \\
\text { Elastase }\end{array}$ & 0.96 \\
\hline Syk Tyr525/526 & $\begin{array}{l}\text { Cannabinoid Rec } \\
2\end{array}$ & 0.91 & Syk Tyr525/526 & $\begin{array}{l}\text { Cannabinoid Rec } \\
2\end{array}$ & 0.86 \\
\hline Syk Tyr525/526 & $\begin{array}{l}\text { РKCaß Thr } \\
638 / 641\end{array}$ & 0.91 & & & \\
\hline Syk Tyr525/526 & CD63 & 0.91 & Syk Tyr525/526 & CD63 & 0.85 \\
\hline Syk Tyr525/526 & CD234 (DARC) & 0.86 & & & \\
\hline Syk Tyr525/526 & DAG Lipase Beta & 0.86 & & & \\
\hline vWF & IL-6 & 0.92 & & & \\
\hline vWF & Syk Tyr525/526 & 0.91 & & & \\
\hline vWF & CD63 & 0.90 & & & \\
\hline vWF & $\begin{array}{l}\text { Cannabinoid Rec } \\
1\end{array}$ & 0.90 & & & \\
\hline vWF & $\begin{array}{l}\text { Neutrophil } \\
\text { Elastase }\end{array}$ & 0.85 & & & \\
\hline
\end{tabular}

TABLE 3: Spearman rho non-parametric pairwise protein correlations quantified in cardioembolic (CE) and large artery atherosclerotic (LAA) thrombus specimens show extensive network 


\section{Cureus}

\section{linkages in CE but not LAA thrombus specimens}

JMP® Statistical Software ver. 5.1, SAS Institute Inc., Cary, USA

The protein linkages (correlations) in CE clot specimens were diverse and more abundant compared to LAA clot specimens (Figure 4). Predominant linkages between PPAR-gamma and arginase-1, CD63, CD234, PKC $\alpha \beta$ Thr 638/641, and von Willebrand factor (vWF) were found in CE clots compared to LAA clots (Table 3). Multiple protein linkages within inflammatory/immune cell proteins arginase-1, neutrophil elastase, CD234, and CD63 were found in CE clot specimens. In contrast, the LAA clots did not have any unique protein-protein correlations compared to the CE clots and lacked significant vWF protein linkages.

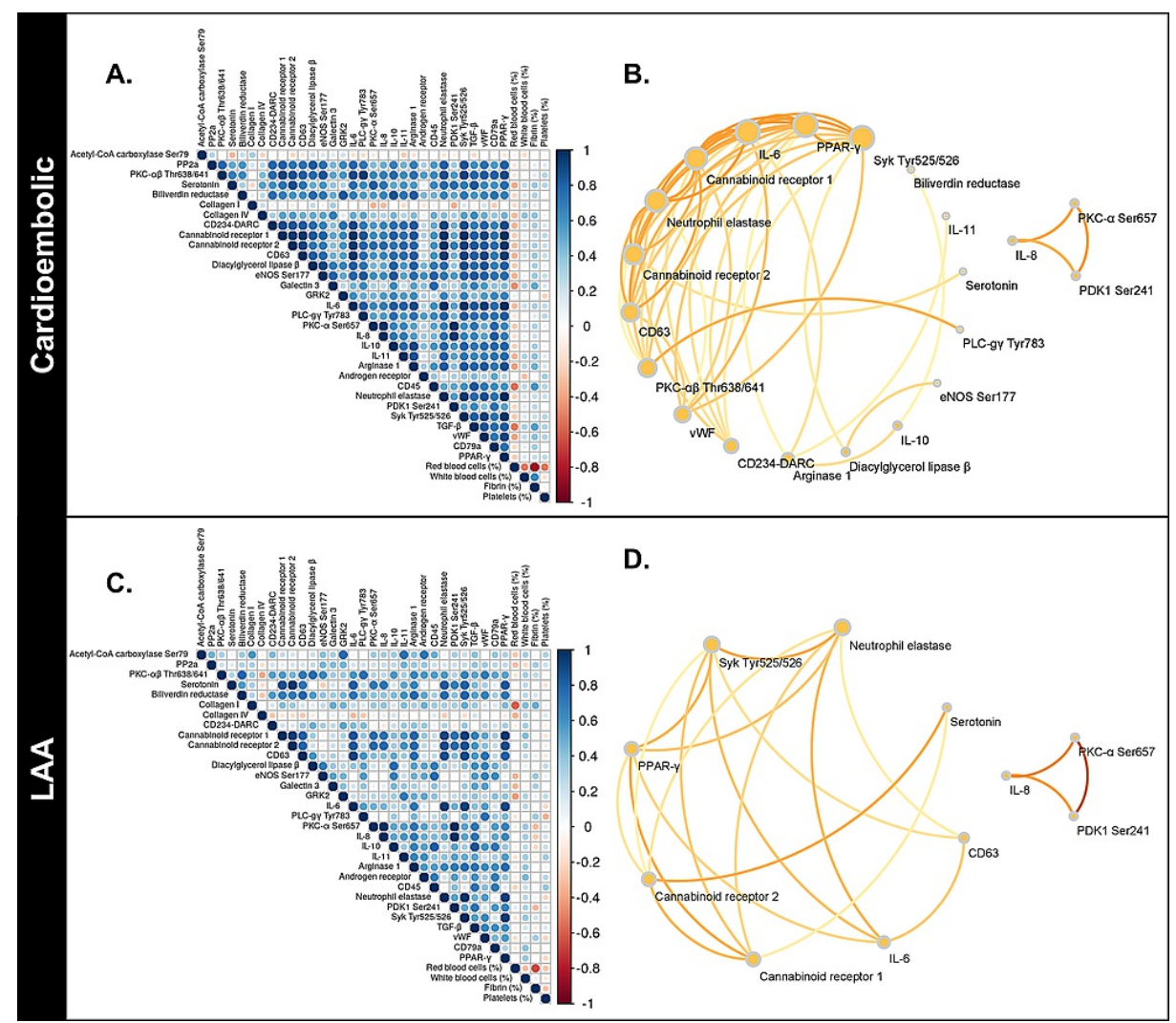

FIGURE 4: Protein interconnections and network signaling differ between thrombus etiology

A correlogram of CE thrombus specimens reveals numerous, strong protein-protein interconnections, shown by the dark blue dots (A). Diverse protein network signaling predominates in CE thrombus specimens (B). In contrast, LAA clots show very few protein-protein interactions (C), with sparse network interconnections (D).

LAA - large artery atherosclerotic; CE - cardioembolic

\section{Discussion}

Our study advances the knowledge of the human cerebrovascular thrombi composition by focusing on the proteomic analysis of cardioembolic and atherothrombotic thrombi. Our results support the general notion that direct analysis of the clots could help in the unveiling, in the future, biomarkers which potentially could assist in stroke diagnosis and differentiating stroke etiologies. Our study comparing the proteomic signatures of CE versus LAA clots demonstrated a number of interesting findings. We found predominant linkages between PPAR-gamma and arginase-1, CD63, CD234, PKC $\alpha \beta$ Thr 638/641, and von Willebrand Factor (vWF) in CE clots suggesting that platelet signaling dominates in CE clots compared to LAA clots. The multiple protein linkages within inflammatory/immune cell proteins lend additional support to the observation of platelet-immune cell cross-talk in CE clot specimens, supported by the white blood cell enrichment seen in the histological analysis of CE emboli in this and previous studies [9]. Proteomic signaling does not necessarily correspond with cellular proportions on histological staining, but it does reflect activated pathways and intra- and inter-cellular communications present in the occlusive clot, influenced by the etiology. Detailed analysis including individual patient histories and medications will 
likely demonstrate strong correlations with certain protein networks, a study we plan to perform with the larger sample currently under investigation.

The widespread use of mechanical thrombectomy devices has resulted in the availability of clot material for histopathological and proteomic analysis. Studies relating histological clot composition to etiology have thus far proved inconclusive [2]. Identifying stroke etiology in this population is very important due to the fact that up to 30\% of large vessel occlusions (LVO) patients do not have a stroke source identified, which complicates secondary stroke prevention strategies. Secondary stroke prevention in this population is essential in preventing recurrent large vessel occlusion, an occurrence that can have a devastating effect on the patient [9]. RPPAs are a powerful tool for assessing proteomic interactions within the cellular microenvironment and may enhance our understanding of the clot formation process in various etiologies, which is why we sought to investigate the proteomic signatures of thrombi from LAA and cardiac etiologies.

Previous proteomics studies have provided some insight, but much more needs to be explored to identify pathways that could aid in differentiating stroke etiology. A previous proteomic study found that two inflammation proteins (integrin alpha-M and mitochondrial superoxide dismutase) are associated with high blood low-density lipoprotein (LDL) [10]. In another study, these two proteins failed to differentiate between cardioembolic vs. atherosclerotic etiology [11]. Also, another study analyzed four thrombi, with $\sim 1,600$ proteins identified [12]. To identify proteomic signatures that can help differentiate between clots of different etiologies such as cardiac and LAA, 31 proteins involved in platelet and/or endothelial function, inflammation, oxidative stress, and metabolism were quantified using reverse phase protein arrays (RPPA) [13-15]. Unsupervised two-way hierarchical clustering revealed two primary clusters of specimens, but despite the difference in etiology between the two thrombus types, the resulting proteomic clusters were quite heterogeneous within each group. However, we did find that platelet signaling dominates in CE clots compared to LAA clots and hope to explore this hypothesis further in larger cohort studies.

Protein signaling networks comprise a series of interconnected proteins and their post-translationally modified forms [6, 16], which are generally organized within specific biological processes, such as inflammation or endothelial integrity. Crosstalk between pathways provides redundancy and resiliency within the cellular microenvironment. Mapping these signaling networks provides functional insights into metabolic processes $[13,16,17]$. The diverse protein signaling interactions found in the clots were determined primarily by analysis of protein phosphorylation cascades. Phosphorylation events are transient functional indicators of active kinase signaling pathways. Specific phosphorylation sites, at any point in time, define the functional character of protein-protein interactions. A set of interacting proteins within an active pathway will tend to be simultaneously phosphorylated in the signaling cascade. Thus, for a given set of patient observations over a disease cohort, correlations among individual protein pairs imply active interactions in a linked pathway $[6,18]$.

\section{Conclusions}

While we demonstrated common protein interactions in thromboembolic disease, consisting of immune, endocannabinoid, and metabolism proteins, CE protein signaling networks are comprised of complex interactions across biological pathways and differ from those seen in LAA patients. Our findings suggest that RPPA of retrieved thrombi in large vessel occlusion could be a promising tool in determining stroke etiology. However, larger studies are needed.

\section{Additional Information \\ Disclosures}

Human subjects: Consent was obtained or waived by all participants in this study. Animal subjects: All authors have confirmed that this study did not involve animal subjects or tissue. Conflicts of interest: In compliance with the ICMJE uniform disclosure form, all authors declare the following: Payment/services info: This work was funded by the National Institutes of Health grant number R01 NS105853. Financial relationships: All authors have declared that they have no financial relationships at present or within the previous three years with any organizations that might have an interest in the submitted work. Other relationships: All authors have declared that there are no other relationships or activities that could appear to have influenced the submitted work.

\section{References}

1. Boodt N, Compagne KCJ, Dutra BG, et al.: Stroke etiology and thrombus computed tomography characteristics in patients with acute ischemic stroke: A MR CLEAN registry substudy. Stroke. 2020, 51:1727-1735. 10.1161/STROKEAHA.119.027749

2. Brinjikji W, Duffy S, Burrows A, et al.: Correlation of imaging and histopathology of thrombi in acute ischemic stroke with etiology and outcome: a systematic review. J Neurointerv Surg. 2017, 9:529-534.

3. Arsava E, Helenius J, Avery R, et al.: Assessment of the predictive validity of etiologic stroke classification . JAMA Neurol. 2017, 74:419-426. 10.1001/jamaneurol.2016.5815

4. Kolominsky-Rabas PL, Weber M, Gefeller O, Neundoerfer B, Heuschmann PU: Epidemiology of ischemic stroke subtypes according to TOAST criteria: incidence, recurrence, and long-term survival in ischemic 
stroke subtypes: a population-based study. Stroke. 2001, 32:2735-2740. 10.1161/hs1201.100209

5. Chiechi A, Mueller C, Boehm KM, et al.: Improved data normalization methods for reverse phase protein microarray analysis of complex biological samples. Biotechniques. 2012, 0:1-7.

6. Petricoin EF, Espina V, Araujo RP, et al.: Phosphoprotein pathway mapping: Akt/mammalian target of rapamycin activation is negatively associated with childhood rhabdomyosarcoma survival. Cancer Res. 2007, 67:3431-3440. 10.1158/0008-5472.CAN-06-1344

7. Signore M, Manganelli V, Hodge A: Antibody validation by western blotting. Methods Mol Biol. 2017, 1606:51-70. 10.1007/978-1-4939-6990-6_4

8. Shannon P, Markiel A, Ozier O, et al.: Cytoscape: a software environment for integrated models of biomolecular interaction networks. Genome Res. 2003, 13:2498-2504. 10.1101/gr.1239303

9. Bouslama M, Haussen DC, Rebello LC, et al.: Repeated mechanical thrombectomy in recurrent large vessel occlusion acute ischemic stroke. Interv Neurol. 2017, 6:1-7. 10.1159/000447754

10. Rao NM, Capri J, Cohn W, et al.: Peptide composition of stroke causing emboli correlate with serum markers of atherosclerosis and inflammation. Front Neurol. 2017, 8:427. 10.3389/fneur.2017.00427

11. Dargazanli C, Zub E, Deverdun J, et al.: Machine learning analysis of the cerebrovascular thrombi proteome in human ischemic stroke: an exploratory study. Front Neurol. 2020, 5:1347. 10.3389/fneur.2020.575376

12. Gallagher RI, Espina V: Reverse phase protein arrays: mapping the path towards personalized medicine. Mol Diagn Ther. 2014, 18:619-630. 10.1007/s40291-014-0122-3

13. Muñoz R, Santamaría E, Rubio I, et al.: Mass spectrometry-based proteomic profiling of thrombotic material obtained by endovascular thrombectomy in patients with ischemic stroke. Int J Mol Sci. 2018, 19:498. 10.3390/ijms 19020498

14. Paweletz CP, Charboneau L, Bichsel VE, et al.: Reverse phase protein microarrays which capture disease progression show activation of pro-survival pathways at the cancer invasion front. Oncogene. 2001, 20:1981-1989. 10.1038/sj.onc.1204265

15. Popova TG, Espina V, Liotta LA, Popov SG: Reverse-phase microarray analysis reveals novel targets in lymph nodes of bacillus anthracis spore-challenged mice. PLoS One. 2015, 10:0129860. 10.1371/journal.pone.0129860

16. Wulfkuhle JD, Berg D, Wolff C, et al.: Molecular analysis of HER2 signaling in human breast cancer by functional protein pathway activation mapping. Clin Cancer Res. 2012, 18:6426-6435. 10.1158/10780432.CCR-12-0452

17. Espina V, Wulfkuhle J, Calvert VS, el al.: Reverse phase protein microarrays for theranostics and patienttailored therapy. Methods Mol Biol. 2008, 441:113-128. 10.1007/978-1-60327-047-2_8

18. Li R, Erdamar S, Dai H, et al.: Forkhead protein FKHR and its phosphorylated form p-FKHR in human prostate cancer. Hum Pathol. 2007, 38:1501-1507. 10.1016/j.humpath.2007.02.016 